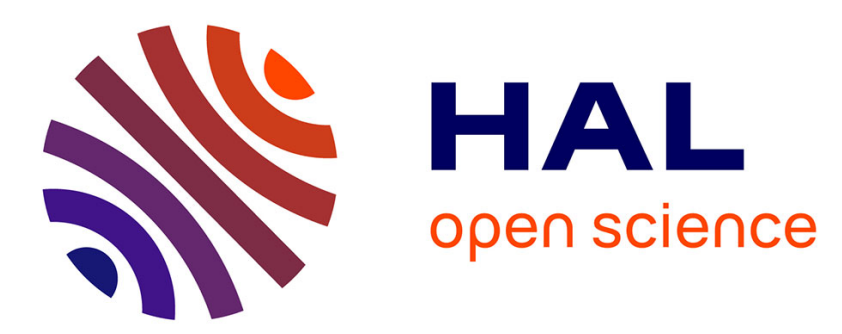

\title{
Nonlinear and Nonseparable Bidimensional Multiscale Representation Based on Cell-Average Representation
}

\author{
Basarab Matei, Sylvain Meignen
}

\section{To cite this version:}

Basarab Matei, Sylvain Meignen. Nonlinear and Nonseparable Bidimensional Multiscale Representation Based on Cell-Average Representation. IEEE Transactions on Image Processing, 2015, 24 (11), pp.4570-4580. 10.1109/TIP.2015.2456424 . hal-01158922

\section{HAL Id: hal-01158922 \\ https://hal.science/hal-01158922}

Submitted on 23 Oct 2015

HAL is a multi-disciplinary open access archive for the deposit and dissemination of scientific research documents, whether they are published or not. The documents may come from teaching and research institutions in France or abroad, or from public or private research centers.
L'archive ouverte pluridisciplinaire $\mathbf{H A L}$, est destinée au dépôt et à la diffusion de documents scientifiques de niveau recherche, publiés ou non, émanant des établissements d'enseignement et de recherche français ou étrangers, des laboratoires publics ou privés. 
Nonlinear and Nonseparable Bidimensional Multiscale Representation Based on

\title{
Cell-Average Representation
}

\author{
Basarab Mateï, \\ LAGA Laboratory, \\ Paris XIII University, France \\ Tel:0033-1-49-40-35-71 \\ FAX:0033-4-48-26-35-68 \\ email: matei@math.univ-paris13.fr \\ Sylvain Meignen* \\ LJK Laboratory, \\ University of Grenoble, France \\ Tel:0033-4-76-51-43-95 \\ email: sylvain.meignen@imag.fr
}

\begin{abstract}
The aim of this paper is to build a new nonlinear and nonseparable multiscale representations of piecewise continuous bidimensional functions. This representation is based on the definition of a linear projection and a nonlinear prediction operator which locally adapts to the function to be represented. This adaptivity of the prediction operator proves to be very interesting for image encoding in that it enables to considerably reduce the number of significant coefficients compared with other representations. Applications of these new nonlinear multiscale representation to image compression and super-resolution conclude the paper.
\end{abstract}

Keywords nonlinear prediction, cell-Average interpolation, image compression, super-resolution 


\section{INTRODUCTION}

For the last decade, research have been carried out to improve multiscale image representation by departing from traditional linear tensor product (bi)orthogonal wavelet representations. In spite these are known not to be optimal in terms of the number of non zero detail coefficients they generate, they are supported by powerful encoders such as EZW [1] or EBCOT [2] and then by optimal quantizers making them very efficient for image compression. Nevertheless, the fact that wavelet representations generate too many detail coefficients has motivated new research toward more compact representations as for instance:

- Frames having some anisotropic directional selectivity, such as curvelets [3] and contourlets [4].

- Bandlets [5] based on tensor products of wavelet bases combined with locally adapted edge operators.

- Edgeprint approximations [6], using wavelet expansions that are computed in the vicinity of an edge, according to a wedge function which locally fits the image.

In all these approaches and in order to take into account the presence of an edge, the multiscale structure is changed. We introduce here a new type of nonlinear multiscale image representation based on cell-average discretization that accurately represents edges with a reduced number of detail coefficients compared with wavelet transforms and keeps the same quadtree structure as the latter. The main difference with respect to wavelet representations is that detail coefficients are computed by means of a local and nonlinear prediction operator.

The new nonlinear multiscale representation (NMR) we introduce in this paper is based on cellaverage discretization and is close to essentially non oscillatory edge adapted (ENO-EA) method which was previously discussed in [7]. First, we recall the general framework for NMR in the cell-average discretization context in Section II. Then, we define a new local and nonlinear prediction operator in Section V that relies both on a novel bidimensional edge detection strategy detailed in Section III and on a new techniques for edge parameters estimation described in Section IV. Numerical simulations showing the compactness of a NMR based on such a prediction operator along with its potential use for image compression and super-resolution conclude the paper.

\section{Harten's Nonlinear Multiscale Representation}

A. Harten introduced in [8] a strategy to construct NMRs based on two interscale discrete operators, called projection and prediction operators respectively denoted by $P_{j-1}^{j}$ and $P_{j}^{j-1}$ in the sequel.

Assuming an image is some function $v$ defined on $[0,1]^{2}$ and $v^{j}$ its approximation on the grid $\left(2^{-j} k_{1}, 2^{-j} k_{2}\right), 0 \leq k_{1}, k_{2} \leq 2^{j}-1$, one first defines a linear projection operator $P_{j-1}^{j}$ acting from 
fine to coarse levels, i.e. , $v^{j-1}=P_{j-1}^{j} v^{j}$. In the cell-average framework this operator is completely characterized since $v_{k}^{j}$ is a rescaled version of a local cell-average of $v$ computed as:

$$
v_{k}^{j}=2^{2 j} \int_{C_{k}^{j}} v(x, y) \mathrm{d} x \mathrm{~d} y,
$$

with $C_{k}^{j}=\left[2^{-j} k_{1}, 2^{-j}\left(k_{1}+1\right)\right] \times\left[2^{-j} k_{2}, 2^{-j}\left(k_{2}+1\right)\right]$, where $k=\left(k_{1}, k_{2}\right)$. In what follows, $C_{k}^{j}$ will be called a cell. From this, one infers that the projection operator reads:

$$
v_{k}^{j-1}=\frac{1}{4}\left(v_{2 k}^{j}+v_{2 k+e_{1}}^{j}+v_{2 k+e_{2}}^{j}+v_{2 k+e_{1}+e_{2}}^{j}\right),
$$

where $e_{1}$ and $e_{2}$ are unit vectors oriented to the right and upward, respectively. The prediction operator $P_{j}^{j-1}$ acts from coarse to fine levels by computing an 'approximation' $\hat{v}^{j}$ of $v^{j}$ from $v^{j-1}$, i.e. $\hat{v}^{j}=$ $P_{j}^{j-1} v^{j-1}$. This operator may be nonlinear. Besides, one assumes that these operators satisfy the following consistency property:

$$
P_{j-1}^{j} P_{j}^{j-1}=I
$$

i.e. the projection of $\hat{v}^{j}$ coincides with $v^{j-1}$ :

$$
v_{k}^{j-1}=\frac{1}{4}\left(\hat{v}_{2 k}^{j}+\hat{v}_{2 k+e_{1}}^{j}+\hat{v}_{2 k+e_{2}}^{j}+\hat{v}_{2 k+e_{1}+e_{2}}^{j}\right) .
$$

The prediction error $e^{j}:=v^{j}-\hat{v}^{j}$ thus satisfies from (3):

$$
P_{j-1}^{j} e^{j}=P_{j-1}^{j} v^{j}-P_{j-1}^{j} \hat{v}^{j}=v^{j-1}-v^{j-1}=0 .
$$

Hence, $e^{j} \in \operatorname{Ker}\left(P_{j-1}^{j}\right)$ and using a basis $E$ of this kernel, one writes $e^{j}$ in a non-redundant way to obtain the detail coefficients $d^{j-1}$ satisfying $e^{j}=E d^{j-1}$. $v^{j}$ is thus completely equivalent to $\left(v^{j-1}, d^{j-1}\right)$. In practice, this non-redundancy means the size of the data is preserved through decomposition. Iterating the proposed nonlinear procedure from the initial data $v^{J}$ (meaning we assume the size of the original image is $2^{J} \times 2^{J}$ ), we obtain its NMR

$$
\mathcal{M} v^{J}=\left(v^{0}, d^{0}, \ldots, d^{J-1}\right)
$$

One says that a prediction operator reproduces bidimensional polynomials of global degree $N$, if $d^{j}=0$ when $v=p$ with $p(x, y)=\sum_{0 \leq i, j \leq N} p_{i, j} x^{i} y^{j}$. Linear and nonlinear prediction operators satisfying polynomial reproduction have been extensively used for image encoding [7]. In the present paper, we are interested in building a new type of nonlinear prediction operator that generate very few detail coefficients when $v$ are some kind of bidimensional piecewise polynomials. 


\section{Edge Detection}

To start with, we shall say that we consider edge detection at level $j-1$ since the prediction at level $j$ is based only on the information available at level $j-1$. In this section, we consider step-edges modeled by straight lines separating regions with constant gray level, that is on a cell $C_{k}^{j-1}$ containing an edge the function $v$ is supposed to have the form

$$
v(x, y)=A \chi_{\{y \geq h(x)\}}(x, y)+B \chi_{\{y<h(x)\}}(x, y),
$$

with $h(x)=m x+n$ and $\chi_{C}(x, y)$ the indicator function of $C, A$ and $B$ being some constants.

The edge detection mechanism makes use of the one dimensional cost functions whose descriptions follow:

$$
\begin{gathered}
H_{k}^{j-1}:=\left|v_{k}^{j-1}-v_{k-e_{1}}^{j-1}\right|+\left|v_{k+e_{1}}^{j-1}-v_{k}^{j-1}\right| \\
V_{k}^{j-1}:=\left|v_{k}^{j-1}-v_{k-e_{2}}^{j-1}\right|+\left|v_{k+e_{2}}^{j-1}-v_{k}^{j-1}\right| .
\end{gathered}
$$

For each $k$ one defines:

$$
\begin{aligned}
& l_{h, k}=\underset{l}{\operatorname{argmin}}\left\{H_{k+l e_{1}}^{j-1}, l \in\{-1,0,1\}\right\} \\
& l_{v, k}=\underset{l}{\operatorname{argmin}}\left\{V_{k+l e_{2}}^{j-1}, l \in\{-1,0,1\}\right\} .
\end{aligned}
$$

As we will use different strategies depending on the orientation of the edge, we need an estimate of the latter. To this end, we define:

$$
\begin{aligned}
& H_{k, 1}^{j-1}=\left(v_{k+e_{2}-e_{1}}^{j-1}+2 v_{k+e_{2}}^{j-1}+v_{k+e_{2}+e_{1}}^{j-1}\right)-\left(v_{k-e_{2}-e_{1}}^{j-1}+2 v_{k-e_{2}}^{j-1}+v_{k-e_{2}+e_{1}}^{j-1}\right) \\
& V_{k, 1}^{j-1}=\left(v_{k+e_{2}-e_{1}}^{j-1}+2 v_{k-e_{1}}^{j-1}+v_{k-e_{2}-e_{1}}^{j-1}\right)-\left(v_{k+e_{2}+e_{1}}^{j-1}+2 v_{k+e_{1}}^{j-1}+v_{k-e_{2}+e_{1}}^{j-1}\right)
\end{aligned}
$$

and then estimate the edge orientation by $m_{k}^{j-1}:=\frac{V_{k, 1}^{j-1}}{H_{k, 1}^{j-1}}$, if $H_{k, 1}^{j-1}$ is non zero and by $\pm \infty$ otherwise, depending on the sign of $V_{k, 1}^{j-1}$. One then considers the set $E^{j-1}$ of cells $C_{k}^{j-1}$ satisfying either:

$$
\begin{gathered}
\text { a) } l_{h, k-e_{1}}=-1 \text { and } l_{h, k+e_{1}}=1 \\
\text { b) } H_{k}^{j-1}>H_{k-e_{1}}^{j-1} \text { and } H_{k}^{j-1}>H_{k+e_{1}}^{j-1} \text {, } \\
\text { c) } m_{k}^{j-1}>1
\end{gathered}
$$


Or

$$
\begin{array}{cc}
\text { a) } & l_{v, k-e_{2}}=-1 \text { and } l_{v, k+e_{2}}=1 \\
\text { b) } & V_{k}^{j-1}>V_{k-e_{2}}^{j-1} \text { and } V_{k}^{j-1}>V_{k+e_{2}}^{j-1} \text {, } \\
\text { c) } & m_{k}^{j-1} \leq 1
\end{array}
$$

This means that when the slope of the edge is estimated to be larger than 1 the edge detection is performed horizontally and vertically otherwise. In the case of the step-edge model described by (6), it is easy to

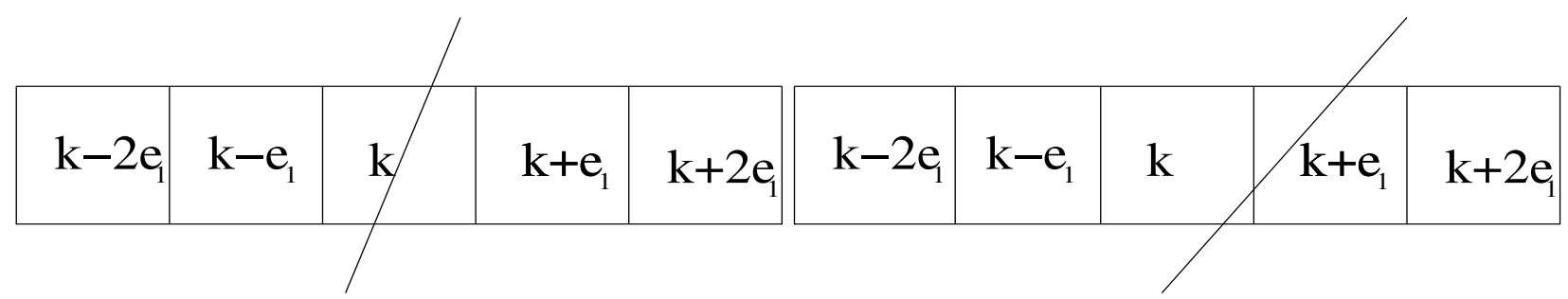

A

B

Fig. 1. A: step-edge crossing cell $C_{k}^{j-1}$; B: step-edge crossing cells $C_{k}^{j-1}$ and $C_{k+e_{1}}^{j-1}$.

check that a cell containing an edge actually satisfies properties (11) a) or (12) a), depending on the slope of the edge. As an illustration of property (11) a), we display on Figure 1 a step-edge crossing either one or two successive cells. In such cases, $l_{h, k-e_{1}}=-1$ and $l_{h, k+e_{1}}=1$ because the cell-average differences are smaller when one moves away from the step-edge. It is worth noting here that when the edge crosses three or more successive cells horizontally, (12) is used insted because then $m_{k}^{j-1}$ is smaller than 1. Furthermore, requirement (11) b) enables to localize the edge on a single cell as we now prove. Without loss of generality, we consider an edge with a slope larger than one crossing $C_{k}^{j-1}$ then we have the following result:

Lemma 3.1: Assume that $v$ is a step-edge function with amplitude $\delta$ as defined in (6) with a slope larger than one crossing $C_{k}^{j-1}$. If among $C_{k-e_{1}}^{j-1}, C_{k}^{j-1}, C_{k+e_{1}}^{j-1}$ only $C_{k}^{j-1}$ is actually crossed by the edge, then only $C_{k}^{j-1}$ belongs to $E^{j-1}$ otherwise if two cells among $C_{k-e_{1}}^{j-1}, C_{k}^{j-1}$, or $C_{k+e_{1}}^{j-1}$ are crossed by the edge then again only one of these cells belongs to $E^{j-1}$.

Proof: We consider two different cases separately:

Case 1. Only $C_{k}^{j-1}$ is crossed by the edge. Since we have

$$
H_{k}^{j-1}=\left|v_{k+e_{1}}^{j-1}-v_{k}^{j-1}\right|+\left|v_{k}^{j-1}-v_{k-e_{1}}^{j-1}\right|=\delta,
$$


and

$$
\left|v_{k+2 e_{1}}^{j-1}-v_{k+e_{1}}^{j-1}\right|=0 \text { or }\left|v_{k-e_{1}}^{j-1}-v_{k-2 e_{1}}^{j-1}\right|=0 .
$$

It follows that $H_{k-e_{1}}^{j-1}<H_{k}^{j-1}$ and $H_{k}^{j-1}>H_{k+e_{1}}^{j-1}$. Now, since $H_{k-2 e_{1}}^{j-1}=0$ and $H_{k+2 e_{1}}^{j-1}=0, l_{h, k-e_{1}}=-1$ and $l_{h, k+e_{1}}=1$, which means that $C_{k}^{j-1}$ belongs to $E^{j-1}$.

Case 2. Cells $C_{k}^{j-1}$ and $C_{k+e_{1}}^{j-1}$ are crossed by the edge. Then, we have $H_{k-e_{1}}^{j-1}<H_{k}^{j-1}$ and $H_{k+2 e_{1}}^{j-1}<$ $H_{k+e_{1}}^{j-1}$. When $H_{k}^{j-1}>H_{k+e_{1}}^{j-1}$, then $l_{h, k-e_{1}}=-1$ and $l_{h, k+e_{1}}=1$, meaning that $C_{k}^{j-e_{1}}$ is an $E^{j-1}$ cell else if $H_{k+e_{1}}^{j-1}<H_{k}^{j-1}, C_{k+e_{1}}^{j-1}$ belongs to $E^{j-1}$. The same reasoning could be made when the two cells crossed by the edge are $C_{k-e_{1}}^{j-1}$ and $C_{k}^{j-1}$.

Note that we could have written a similar lemma assuming the edge slope to be smaller than one. On the contrary, if we used only (11) a) an c) then for cases corresponding to Figure $1 \mathrm{~B}$, the edge could be localized on two successive cells.

Finally, from the set $E^{j-1}$ we define the subset:

$\mathcal{E}^{j-1}=\left\{C_{k}^{j-1} \in E^{j-1}, C_{k-e_{1}+e_{2}}^{j-1}, C_{k+e_{1}-e_{2}}^{j-1} \notin E^{j-1}\right.$ if $m_{k}^{j-1}>0, C_{k+e_{1}+e_{2}}^{j-1}, C_{k-e_{1}-e_{2}}^{j-1} \notin E^{j-1}$ if $\left.m_{k}^{j-1}<0\right\}$

Considering

$$
\begin{aligned}
& E_{V}^{j-1}=\left\{C_{k}^{j-1} \in \mathcal{E}^{j-1} \text { s. t. }\left|m_{k}^{j-1}\right| \geq 1\right\} \\
& E_{H}^{j-1}=\left\{C_{k}^{j-1} \in \mathcal{E}^{j-1} \text { s. t. }\left|m_{k}^{j-1}\right|<1,\right\} .
\end{aligned}
$$

we have $\mathcal{E}^{j-1}=E_{H}^{j-1} \cup E_{V}^{j-1}$ which we call the set of edge-cells in what follows. An illustration of the extra constraint put on $E^{j-1}$ to obtain $\mathcal{E}^{j-1}$ is shown on Figure $2 \mathrm{~A}$, when $m_{k}^{j-1}>0$. We need to remark that to define $E^{j-1}$ as we did is crucial since if it were defined using only (11) a) and c) or (12) a) and c) false detections create holes in the set $\mathcal{E}^{j-1}$ where there should be a continuum of cells as shown on Figure $2 \mathrm{C}$. This does not happen when the $\mathcal{E}^{j-1}$ is defined using (11) a)-c) or (12) a)-c) (see Figure 2 D). Finally, we also notice that adding conditions (11) b) and (12) b) improves the robustness of the detection since we get much less false detections inside smooth regions (compare again Figure 2 $\mathrm{C}$ and $\mathrm{D})$. By using this new edge detector, we remove some false detections but also some real ones ; we will see later how to recover the latter.

\section{Estimation of Edge Parameters and Definition of S-CELls}

We assume in this section that $C_{k}^{j-1}$ is an edge-cell and we consider the more general step-edge model for function $v$ inside $C_{k}^{j-1}$, i.e.

$$
v(x, y):=a(x, y) \chi_{D}(x, y)+b(x, y) \chi_{D^{c}}(x, y),
$$




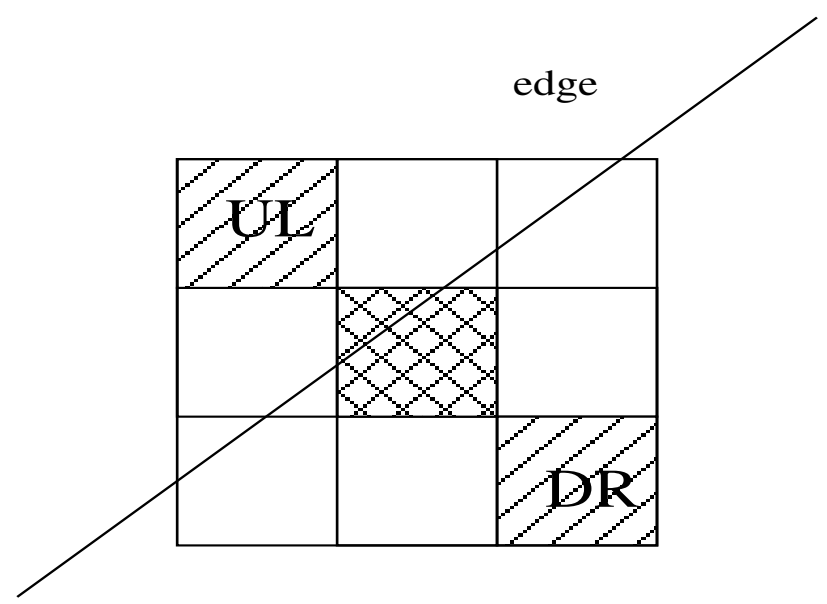

A

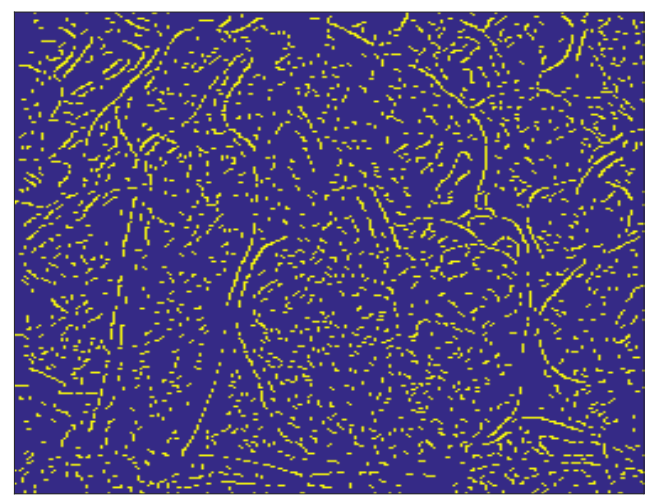

$\mathrm{C}$

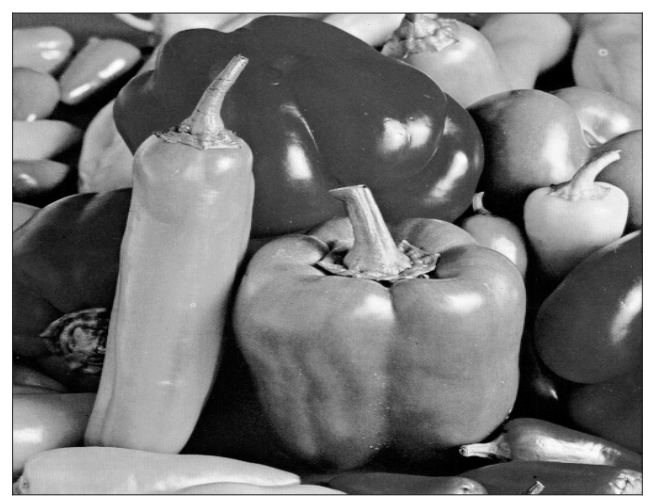

B

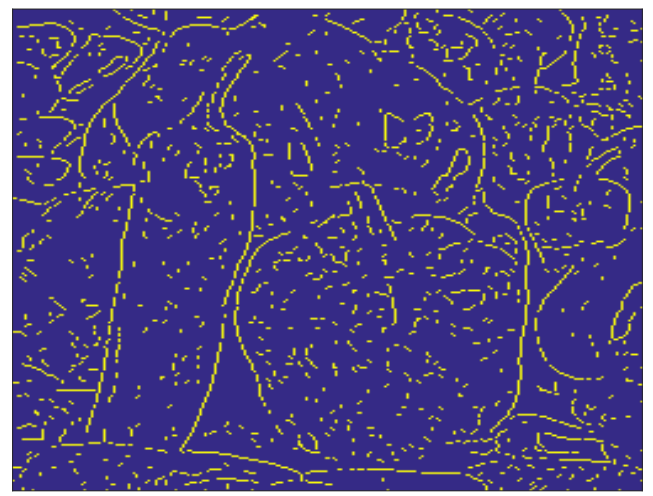

$\mathrm{D}$

Fig. 2. A: illustrates how the set $\mathcal{E}^{j-1}$ is built when $m_{k}^{j-1}>0$, the cells denoted by $U L$ (for upper left) and $D R$ (for down right) should not belong to $E^{j-1}$; B: $512 \times 512$ image of peppers; C: cells in $\mathcal{E}^{j-1}$ computed at level $J-1$ from image B using only (11) a) and c) or (12) a) and c) at level $J-1$; D: idem as C but using (11) a)-c) or (12) a)-c) to compute edge-cells.

where $D=\{(x, y), y \geq h(x)\}$ and $D^{c}=C_{k}^{j-1} \backslash D . h(x)$ will either be approximated by a straight line $p_{1, k}(x)=l x+q$ or by a second order polynomial $p_{2, k}(x)=m x^{2}+n x+p$, where index $k$ indicates the polynomial is different at each location $k$. As for $a(x, y)$ and $b(x, y)$, they will either be approximated by constants $A$ and $B$ or by biquadratic polynomials $p_{a, k}$ and $p_{b, k}$, i.e. $p_{a, k}(x, y)=\sum_{0 \leq i, q \leq 2} a_{i, q} x^{i} y^{q}\left(p_{b, k}\right.$ being defined similarly from coefficients $b_{i, q}$ ). We will see later in which instances the analysis benefits from considering a curve model for the edge and non constant $p_{a, k}$ and $p_{b, k}$. Regarding the edge model, we shall say that the approach proposed in [7], subsequently denoted by ENO-EA, involves constant approximations for $a(x, y)$ and $b(x, y)$ and edges locally modeled by straight lines. Nevertheless, this 
model is only valid when the scale of study $2^{-j+1}$ is lower than some critical scale $h_{c}$ whose definition is now recalled for the sake of consistency [7]. Let $v$ be a piecewise smooth function containing a curved edge $I$. For a cell $C_{k}^{j-1}$ crossed by $I$ one defines its neighborhood $D_{k}^{j-1}$ by

$$
D_{k}^{j-1}:=\left\{C_{k+m e_{1}+n e_{2}}^{j-1}, m, n \in\{-1,0,1\}\right\},
$$

and given an arclength parameterization $\gamma(t)$ of $I$, one considers

$$
I_{k}^{j-1}=\left\{t ; \gamma(t) \in D_{k}^{j-1}\right\},
$$

the set of values $t$ for which $I$ crosses $D_{k}^{j-1}$. Following [7], the critical scale of detection is given by

$$
\frac{1}{h_{c}}:=\frac{\sup _{D_{k}^{j-1} \backslash I}\|\nabla v(x, y)\|}{\sup _{I_{k}^{j-1}}\|[v](t)\|}+\sup _{I_{k}^{j-1}}\left|\gamma^{\prime \prime}(t)\right|,
$$

where $\|[v](t)\|$ is the amplitude of the step at $\gamma(t)$. Our goal is to show that, as in practical situations such as image analysis one cannot fix the scale of study to a value smaller than $h_{c}$, it is worth considering more complex edge models than straight lines. This aspect will become clearer in the numerical section.

\section{A. Estimation of Edge Parameters with ENO-EA Method}

In ENO-EA method, function $h(x)$ is estimated by an affine polynomial $p_{1, k}$ computed on $C_{k}^{j-1}$ belonging to $E_{V}^{j-1}$ and assuming $m_{k}^{j-1}$ is positive as follows (the same kind of computation can easily be transposed to any other cases). To estimate $h(x)$ one needs two different points which are obtained using the following arguments. Assuming $C_{k-2 e_{1}}^{j-1}$ and $C_{k+2 e_{1}}^{j-1}$ are not crossed by an edge, one has the following consistency property:

$$
v_{k-e_{1}}^{j-1}+v_{k}^{j-1}+v_{k+e_{1}}^{j-1}=x_{0} v_{k-2 e_{1}}^{j-1}+\left(3-x_{0}\right) v_{k+2 e_{1}}^{j-1} .
$$

To explain where this consistency property comes from, we refer to Figure $3 \mathrm{~A}$ on which $y_{0}=\frac{1}{2}$ so that the two hatched areas filled with dotted lines are equal. This implies that the normalized integral of $v$ on the union of $C_{k-e_{1}}^{j-1}, C_{k}^{j-1}$ and $C_{k+e_{1}}^{j-1}$, i.e. $v_{k-e_{1}}^{j-1}+v_{k}^{j-1}+v_{k+e_{1}}^{j-1}$, is equal to $x_{0} v_{k-2 e_{1}}^{j-1}+\left(3-x_{0}\right) v_{k+2 e_{1}}^{j-1}$ because, in ENO-EA method, $v$ is supposed to be piecewise constant on each side of the edge, i.e. $v_{k-2 e_{1}}^{j-1}=A$ and $v_{k+2 e_{1}}^{j-1}=B$. So, in that framework and taking into account the scale factor, we get that the edge passes through $\left(\frac{k_{1}-1+x_{0}}{2^{j-1}}, \frac{k_{2}+1 / 2}{2^{j-1}}\right)$. Now, we remark that since $m_{k}^{j-1}>1$, the edge crossing $C_{k}^{j-1}$ can only cross, on the row indexed by $k_{2}+1, C_{k+e_{2}}^{j-1}, C_{k+e_{1}+e_{2}}^{j-1}$ and $C_{k+2 e_{1}+e_{2}}^{j-1}$ and, on the row indexed by $k_{2}-1, C_{k-2 e_{1}-e_{2}}^{j-1}, C_{k-e_{1}-e_{2}}^{j-1}$ and $C_{k-e_{2}}^{j-1}$ (see Figure $3 \mathrm{~B}$ ). We define two points $N$ and $P$ using consistency rules of type (19) on rows indexed by $k_{2}+1$ and $k_{2}-1$ respectively. $h(x)$ is then defined as the straight line passing through $N$ and $P$. Also, with this method, one considers $p_{a, k}(x, y)=v_{k-e_{1}+e_{2}}^{j-1}$ and $p_{b, k}(x, y)=v_{k+e_{1}-e_{2}}^{j-1}$ (the situation being summarized on Figure $3 \mathrm{~B}$ ). 


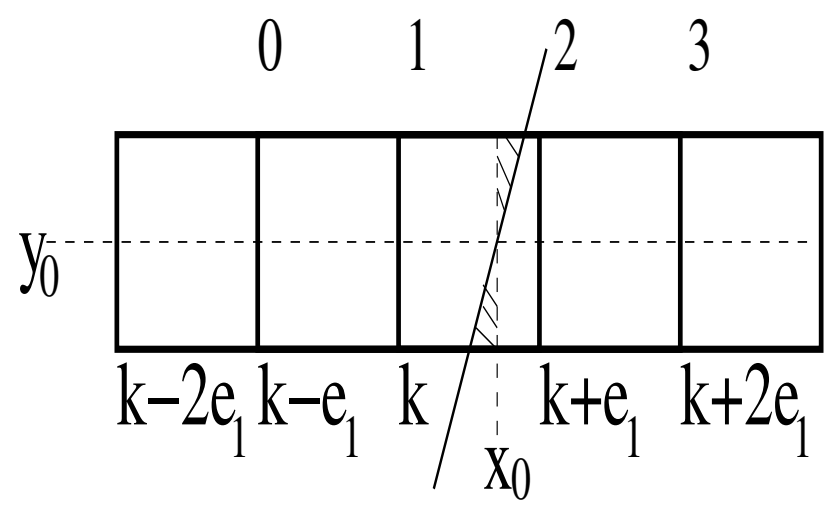

A

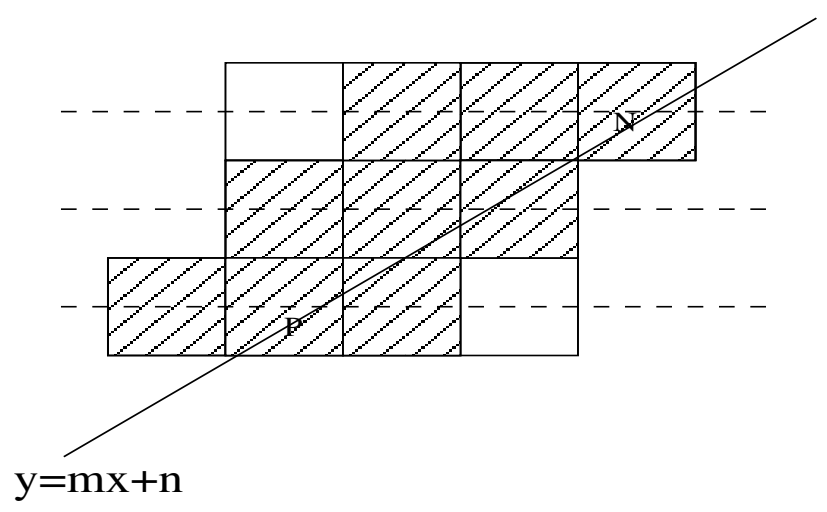

B

Fig. 3. A: Illustration of the consistency property on the rows, the digits above the figure correspond to the scale for $x_{0}$; B: Cells involved in the computation of edge parameters along with actual edge.

\section{B. New Estimation of Edge Parameters}

In the following sections we will make extensive used of the unique biquadratic polynomial $p_{k}$ interpolating the average of $v$ on the cells making up $D_{k}^{j-1}$ and defined as follows:

$$
v_{k+l e_{1}+p e_{2}}^{j-1}=2^{2(j-1)} \int_{C_{k+l e_{1}+p e_{2}}^{j-1}} p_{k}(x, y) \mathrm{d} x \mathrm{~d} y \quad l, p \in\{-1,0,1\} .
$$

1) Computation of polynomials $p_{a, k}(x, y)$ and $p_{b, k}(x, y)$ : we here explain how we compute $p_{a, k}(x, y)$ and $p_{b, k}(x, y)$ ) on $C_{k}^{j-1}$ in $E_{V}^{j-1}$ assuming $m_{k}^{j-1}$ is positive (the same kind of computation could be carried out in the other cases). We first consider that the studied cell is such that (see Figure 4 A for an illustration):

$$
\left(D_{k+2\left(e_{2}-e_{1}\right)}^{j-1} \cup D_{k+2\left(e_{1}-e_{2}\right)}^{j-1}\right) \cap \mathcal{E}^{j-1}=\emptyset .
$$

If (21) is satisfied, polynomials $p_{a, k}$ and $p_{b, k}$ are respectively defined as $p_{k-2 e_{1}+2 e_{2}}$ and $p_{k+2 e_{1}-2 e_{2}}$ (where $p_{k}$ was introduced in (20)). Note that if $a(x, y)$ and $b(x, y)$ are actually biquadratic polynomials on each side of the edge then $p_{a, k}=a$ and $p_{b, k}=b$. When (21) is not satisfied, we put $p_{a, k}(x, y)=A$ and $p_{b, k}(x, y)=B$, with $A=v_{k-e_{1}+e_{2}}^{j-1}$ and $B=v_{k+e_{1}-e_{2}}^{j-1}$.

2) Estimation of $h(x)$ : to estimate $h(x)$ when it is modeled by $p_{2, k}(x)$ on $C_{k}^{j-1}$ in $E_{V}^{j-1}$, still assuming $m_{k}^{j-1}$ is positive, we define three points $N, M$ and $P$ on the rows indexed by $k_{2}+1, k_{2}$ and $k_{2}-1$ respectively by using a generalization of rule (19). It is important to remark here that, by analogy, points $N, M$ and $P$ could be computed using consistency property of type (19) on the columns indexed by $k_{1}-1, k_{1}$ and $k_{1}+1$ for cells in $E_{H}^{j-1}$ (this can be viewed as the transposed situation to the studied case). 
Let us write down the generalization of the consistency rule (19) on the row indexed by $k_{2}$. We first integrate polynomials $p_{a, k}$ and $p_{b, k}$ with respect to $y$ to define:

$$
A_{q, k}^{j-1}(x):=\int_{\frac{q}{2^{j-1}}}^{\frac{q+1}{2^{j-1}}} p_{a, k}(x, y) \mathrm{d} y, B_{q, k}^{j-1}(x):=\int_{\frac{q}{2^{j-1}}}^{\frac{q+1}{2^{j-1}}} p_{b, k}(x, y) \mathrm{d} y,
$$

and then compute the abscissa $x_{M}$ of $M$ through the following consistency rule:

$$
v_{k-e_{1}}^{j-1}+v_{k}^{j-1}+v_{k+e_{1}}^{j-1}=L_{k_{1}, k_{2}}^{j-1}\left(x_{M}\right)
$$

where:

$$
L_{k_{1}, k_{2}}^{j-1}\left(x_{M}\right):=2^{2(j-1)}\left(\int_{\frac{k_{1}-1}{2^{j-1}}}^{\frac{k_{1}-1+x_{M}}{2^{j-1}}} A_{k_{2}, k}^{j-1}(x) d x+\int_{\frac{k_{1}-1+x_{M}}{2^{j-1}}}^{\frac{k_{1}+2}{2^{j-1}}} B_{k_{2}, k}^{j-1}(x) d x\right) .
$$

From Equation (23), $x_{M}$ is a root on $[0,3]$ of a third order polynomial. The motivation for integrating biquadratic polynomials $p_{k, a}$ and $p_{k, b}$ in the vertical direction is the remark that in images the grey level often varies smoothly along the edge. Also, this enables us to simplify the search for point $M$ into a one-dimensional problem. Indeed, we again define $y_{M}:=\frac{k_{2}+1 / 2}{2^{j-1}}$ and use the same arguments as in the piecewise constant case (see Section IV-A) to justify this new consistency rule. This means that point $M$ is not on the edge curve but sufficiently close to it to bring significant improvement in terms of edge estimation compared with a straight line estimation as we will see in the numerical section.

Then, similarly to what was done in the ENO-EA case, if one assumes the slope of the edge does not vary too rapidly along the vertical direction, potential edge-cells on the row indexed by $k_{2}+1$ (resp. $\left.k_{2}-1\right)$ are $C_{k+e_{2}}^{j-1}, C_{k+e_{1}+e_{2}}^{j-1}$ and $C_{k+2 e_{1}+e_{2}}^{j-1}$ (resp. $C_{k-2 e_{1}-e_{2}}^{j-1}, C_{k-e_{1}-e_{2}}^{j-1}$ and $C_{k-e_{2}}^{j-1}$, see Figure 4 B for an illustration). Consistency rule (23) on rows indexed by $k_{2}+1$ and $k_{2}-1$ then respectively read

$$
\begin{aligned}
& v_{k+e_{2}}^{j-1}+v_{k+e_{2}+e_{1}}^{j-1}+v_{k+e_{2}+2 e_{1}}^{j-1}=L_{k_{1}+1, k_{2}+1}^{j-1}\left(x_{N}\right) \\
& v_{k-e_{1}-2 e_{2}}^{j-1}+v_{k-e_{2}-e_{1}}^{j-1}+v_{k-e_{2}}^{j-1}=L_{k_{1}-1, k_{2}-1}^{j-1}\left(x_{P}\right),
\end{aligned}
$$

so that both $x_{N}$ and $x_{P}$ are roots of third order polynomials on $[0,3]$ and $y_{N}=\frac{k_{2}+3 / 2}{2^{j-1}}$ while $y_{P}=\frac{k_{2}-1 / 2}{2^{j-1}}$. We shall finally stress that to define all these consistency rules, we use polynomials $p_{a, k}$ and $p_{b, k}$ on each of the rows indexed by $k_{2}-1, k_{2}$ and $k_{2}+1$.

When (21) is not satisfied, to take into account a potential variation of the gray level in the direction of the edge, we adopt the following strategy:

- $A_{k_{2}+1, k}^{j-1}(x)$ is replaced by $2^{-2(j-1)} v_{k-e_{1}+e_{2}}^{j-1}$ and $B_{k_{2}+1, k}^{j-1}(x)$ by $2^{-2(j-1)} v_{k+3 e_{1}+e_{2}}^{j-1}$ in $L_{k_{1}+1, k_{2}+1}\left(x_{N}\right)$ provided $C_{k-e_{1}+e_{2}}^{j-1}, C_{k+3 e_{1}+e_{2}}^{j-1} \notin \mathcal{E}^{j-1}$.

- $A_{k_{2}, k}^{j-1}(x)$ is replaced by $2^{-2(j-1)} v_{k-2 e_{1}}^{j-1}$ and $B_{k_{2}, k}^{j-1}(x)$ by $2^{-2(j-1)} v_{k+2 e_{1}}^{j-1}$ in $L_{k_{1}, k_{2}}\left(x_{M}\right)$ provided $C_{k-2 e_{1}}^{j-1}, C_{k+2 e_{1}}^{j-1} \notin \mathcal{E}^{j-1}$. 
- $A_{k_{2}-1, k}^{j-1}(x)$ is replaced by $2^{-2(j-1)} v_{k-3 e_{1}-e_{2}}^{j-1}$ and $B_{k_{2}-1, k}^{j-1}(x)$ by $2^{-2(j-1)} v_{k+e_{1}-e_{2}}^{j-1}$ in $L_{k_{1}-1, k_{2}-1}\left(x_{P}\right)$ provided $C_{k-3 e_{1}-e_{2}}^{j-1}, C_{k+e_{1}-e_{2}}^{j-1} \notin \mathcal{E}^{j-1}$.

Note that this framework is easily transposable to $E_{V}^{j-1}$ cells associated with negative $m_{k}^{j-1}$. Indeed, the same reasoning as previously could be carried out remarking the cells potentially containing an edge in that case are those indicated on Figure $4 \mathrm{C}$ (assuming the central cell is $C_{k}^{j-1}$ ).

While trying to compute edge parameters we determine a subset $\tilde{\mathcal{E}}^{j-1}$ of $\mathcal{E}^{j-1}$ corresponding to the edge-cells for which the parameters are actually computable, so that $\mathcal{E}^{j-1}=\tilde{\mathcal{E}}^{j-1} \cup \mathcal{D}^{j-1}$. It is worth remarking here that T-junctions are edges with locally high curvature will be contained in the set $\mathcal{D}^{j-1}$ because these edge cells are typically outside the proposed step-edge model.

\section{Definition of S-cells}

In our formalism, $S$-cells are going to be the cells actually crossed by an edge for which the computation of the edge parameters is possible. The set of S-cells will definitely contain $\mathcal{E}^{j-1}$ but much more cells are crossed by an edge as explained earlier. To build the set of S-cells, we proceed iteratively starting from $\tilde{\mathcal{E}}^{j-1}$ and using the parameters of the edge computed on the cells making up that set. To explain how we proceed, we consider the case of a cell $C_{k}^{j-1}$ in $\tilde{\mathcal{E}}^{j-1} \cap E_{V}^{j-1}$. As already noticed above, the studied edge can also potentially cross cells $C_{k-e_{1}}^{j-1}$ and $C_{k+e_{1}}^{j-1}$. One then just checks whether the edge estimated on cell $C_{k}^{j-1}$ crosses one of the latter. If so, one tries to recompute the parameters of the edge on this cell and if it is successful this cell is added to the set of S-cells. The same kind of procedure is applied to the set of cells $\tilde{\mathcal{E}}^{j-1} \cap E_{H}^{j-1}$ for which we check whether the edge computed on $C_{k}^{j-1}$ crosses $C_{k-e_{2}}^{j-1}$ or $C_{k+e_{2}}^{j-1}$.

Finally, because the addition of new S-cells is based only on a estimate of the edge parameters, we may add cells in such a way that some S-cells $C_{k}^{j-1}$ such that $m_{k}^{j-1}>0$ have neighbors $C_{k-e_{1}+e_{2}}^{j-1}$ and $C_{k+e_{1}-e_{2}}^{j-1}$ also considered as S-cells. To do away with this cases and when $m_{k}^{j-1}>1$ we keep $C_{k}^{j-1}$ in the set of S-cells if $H_{k}^{j-1}>H_{k-e_{1}+e_{2}}^{j-1}$ and $H_{k}^{j-1}>H_{k+e_{1}-e_{2}}^{j-1}$ and when $m_{k}^{j-1}<1$, we keep $C_{k}^{j-1}$ in the set if $V_{k}^{j-1}>V_{k-e_{1}+e_{2}}^{j-1}$ and $V_{k}^{j-1}>V_{k+e_{1}-e_{2}}^{j-1}$. The same kind of reasoning is made when $m_{k}^{j-1}$ is negative.

\section{Definition of the Nonlinear Prediction Operator}

Once we have determined S-cells, the other cells are automatically labelled by O. In the following sections, we detail how we build the prediction operator knowing the nature of the cells and their corresponding edge parameters (for S-cells). 


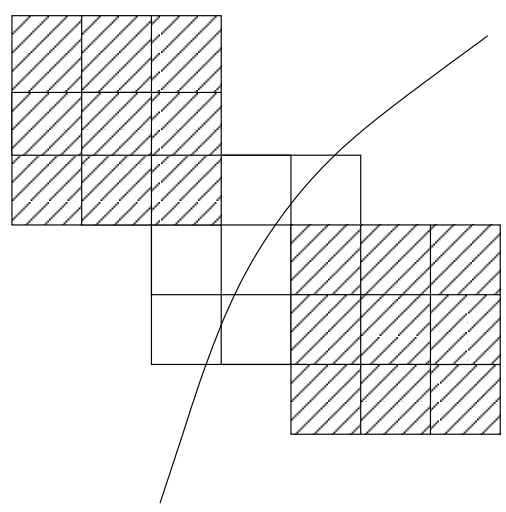

A

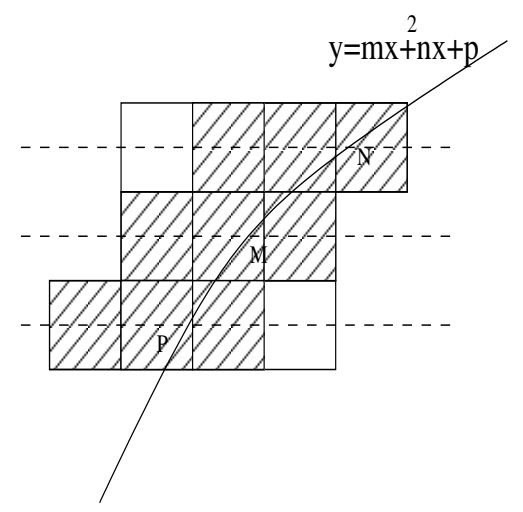

B

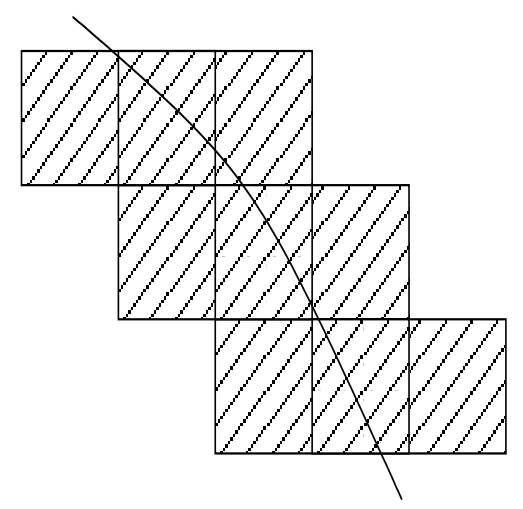

$\mathrm{C}$

Fig. 4. A: The upper and lower hatched regions represent the neighborhood $D_{k-e_{1}+e_{2}}^{j-1}$ and $D_{k+e_{1}-e_{2}}^{j-1}$ respectively used to compute $p_{a, k}$ and $p_{b, k}$ when condition (21) is satisfied; B: points $M, N, P$ along with the polynomial $p_{2, k}(x)=m x^{2}+n x+p$ interpolating these points, the hatched zone corresponds to the region potentially crossed by the edge; C: The hatched region corresponds to the cells that may be intersected by the edge when $m_{k}^{j-1}$ is smaller than -1 .

\section{A. Prediction on $O$-cells}

To build the prediction operator on $C_{k}^{j-1}$ supposed to be an O-cell, we use a biquadratic polynomial $p_{k^{*}}$ approximating function $v$ which is built by interpolation of the average of $v$ on the cells making up stencil $D_{k^{*}}^{j-1}$. To find the latter, ENO-EA method needs the definition of $F_{k}^{j-1}=H_{k, 2}^{j-1}+V_{k, 2}^{j-1}$ where

$$
\begin{aligned}
H_{k, 2}^{j-1} & :=\sum_{p=-1}^{1}\left|v_{k-e_{1}+p e_{2}}^{j-1}-v_{k+p e_{2}}^{j-1}\right|+\left|v_{k+p e_{2}}^{j-1}-v_{k+e_{1}+p e_{2}}^{j-1}\right| \\
V_{k, 2}^{j-1} & :=\sum_{l=-1}^{1}\left|v_{k+l e_{1}-e_{2}}^{j-1}-v_{k+l e_{1}}^{j-1}\right|+\left|v_{k+l e_{1}}^{j-1}-v_{k+l e_{1}+e_{2}}^{j-1}\right|,
\end{aligned}
$$

which corresponds to the sum of all the moduli of the first order vertical and horizontal finite differences on the stencil $D_{k}^{j-1}$. ENO-EA method [9] finds $k^{*}$ associated with the least oscillatory $3 \times 3$ stencil containing $C_{k}^{j-1}$, i.e.:

$$
k^{*}=\underset{q}{\operatorname{argmin}}\left\{F_{q}^{j-1}, q=k+l e_{1}+p e_{2} l, p \in\{-1,0,1\}\right\} .
$$

However, it is well known that this method for stencil selection is particularly unstable since a slight change in the data may entail a change of stencil. To overcome this problem, we put $k^{*}=k$ when $D_{k}^{j-1}$ does not contain a S-cell. In other cases, rather than using the cost function $F_{k}^{j-1}$, we preferentially use the following strategy that minimizes the displacement with respect to the centered stencil: 
- Find $k^{*}$ associated with the first (if any) stencil in the list $\left\{D_{k-e_{2}}^{j-1}, D_{k+e_{1}}^{j-1}, D_{k+e_{2}}^{j-1}, D_{k-e_{1}}^{j-1}\right\}$ not containing any S-cells.

- If no stencil is selected by the above process, find $k^{*}$ associated with the first (if any) stencil in the list $\left\{D_{k+e_{1}+e_{2}}^{j-1}, D_{k+e_{1}-e_{2}}^{j-1}, D_{k-e_{1}-e_{2}}^{j-1}, D_{k-e_{1}+e_{2}}^{j-1}\right\}$ not containing any S-cells.

If the above mechanism does not find any suitable stencil, we again put $k^{*}=k$. By using such a procedure based on the location of S-cells instead of $F_{k}^{j-1}$, we no longer use a cost function to determine the stencils we use for prediction on O-cells. Note also that to choose the stencil $D_{k^{*}}^{j-1}$ for prediction, means that we define polynomial $p_{k^{*}}$ to predict that is:

$$
\hat{v}_{2 k+l e_{1}+q e_{2}}^{j}=2^{2 j} \int_{C_{2 k+l e_{1}+q e_{2}}^{j}} p_{k^{*}}(x, y) \mathrm{d} x \mathrm{~d} y, l, q \in\{0,1\} .
$$

To illustrate this procedure, in the case of a straight line edge, we display on Figure 5 A, S-cells labeled by 3 , O-cells on which stencil $D_{k}^{j-1}$ is used to predict labeled by 1 and by 2 the O-cells on which stencil $D_{k^{*}}^{j-1}$ is used instead.

\section{B. Prediction on S-cells}

We now present the prediction scheme on a S-cell when we model the step-edge by a second order polynomial and the image on each side of the edge by a biquadratic polynomial. We first consider $C_{k}^{j-1} \in \tilde{\mathcal{E}}^{j-1}$ associated with two biquadratic polynomials $p_{a, k}$ and $p_{b, k}$ and an edge equation modeled by second order polynomial $p_{2, k}$. These parameters define an approximation of $v$ inside $C_{k}^{j-1}$ of the form:

$$
p_{S}(x, y):=p_{a, k}(x, y) \chi_{\left\{y \geq p_{2, k}(x)\right\}}(x, y)+p_{b, k}(x, y) \chi_{\left\{y<p_{2, k}(x, y)\right\}}(x, y) .
$$

We then estimate the average at level $j$, on the four subcell of cell $C_{k}^{j-1}$ by:

$$
\hat{v}_{2 k+l e_{1}+q e_{2}}^{j}=2^{2 j} \int_{C_{2 k+l e_{1}+q e_{2}}} p_{S}(x, y) \mathrm{d} x \mathrm{~d} y, l, q \in\{0,1\} .
$$

The prediction based on the piecewise biquadratic polynomial $p_{S}$ is however not strictly speaking consistent with (4) because $p_{2, k}$ is only an estimate of the real edge. Therefore, we force the prediction into being consistent on $C_{k}^{j-1}$ by defining $m_{a}, m_{b}, m_{c}$ and $m_{d}$ the final prediction on the four subcells of $C_{k}^{j-1}$ (as depicted on Figure 5 B) as follows. If $m_{k}^{j-1}$ is positive and if $p_{S}\left(\frac{k_{1}+1 / 2}{2^{j-1}}, \frac{k_{2}+1 / 2}{2^{j-1}}\right)=$ $p_{b, k}\left(\frac{k_{1}+1 / 2}{2^{j-1}}, \frac{k_{2}+1 / 2}{2^{j-1}}\right)$, we are in the situation depicted on Figure $5 \mathrm{~B}$, and we put:

$$
m_{a}:=\hat{v}_{2 k}^{j}, m_{d}:=\hat{v}_{2 k+e_{1}}^{j}, m_{c}:=\hat{v}_{2 k+e_{1}+e_{2}}^{j}, m_{b}=4 v_{k}^{j-1}-\left(m_{a}+m_{b}+m_{c}\right) .
$$


On the contrary, when $p_{S}\left(\frac{k_{1}+1 / 2}{2^{j-1}}, \frac{k_{2}+1 / 2}{2^{j-1}}\right)=p_{a, k}\left(\frac{k_{1}+1 / 2}{2^{j-1}}, \frac{k_{2}+1 / 2}{2^{j-1}}\right)$, we leave $m_{a}, m_{b}$ and $m_{c}$ unchanged and use (4) to define $m_{d}$. The same kind of computation could be made when $m_{k}^{j-1}$ is negative. It is important to remark that when the edge intersects only one of the four subcells, such a procedure enables to compensate for the lack of accuracy in the determination of the location of the edge.

\begin{tabular}{|l|l|l|l|l|l|l|l|}
\hline 1 & 1 & 1 & 2 & 2 & 3 & 3 & 2 \\
\hline 1 & 1 & 2 & 2 & 3 & 3 & 2 & 2 \\
\hline 1 & 2 & 2 & 3 & 3 & 2 & 2 & 1 \\
\hline 2 & 2 & 3 & 3 & 2 & 2 & 1 & 1 \\
\hline 2 & 3 & 3 & 2 & 2 & 1 & 1 & 1 \\
\hline 3 & 3 & 2 & 2 & 1 & 1 & 1 & 1 \\
\hline
\end{tabular}

A

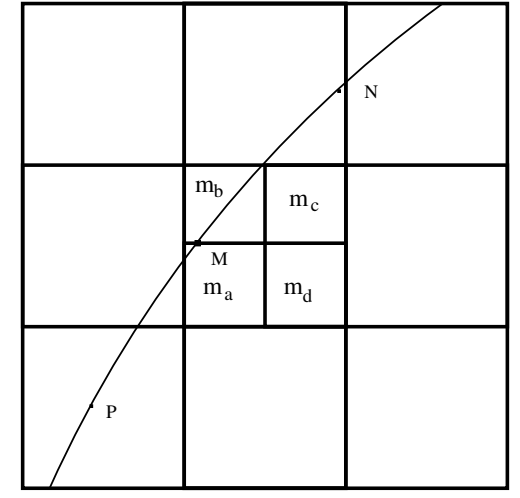

B

Fig. 5. A: illustration in the case of a straight line edge of the different type of cells (3: S-cells, 2: O-cells associated with shifted stencil for prediction, 1: O-cells associated with centered stencil for prediction); B: illustration of the procedure to ensure that the prediction satisfies (4).

\section{Nonlinear Multiscale Representation}

Once a prediction operator has been defined, to obtain a non redundant representation one rewrites the prediction error in a basis of the projection operator. Denoting $e_{k}^{j}:=v_{k}^{j}-\hat{v}_{k}^{j}$, the consistency property satisfied by the prediction operator implies:

$$
e_{2 k}^{j}+e_{2 k-e_{1}}^{j}+e_{2 k-e_{2}}^{j}+e_{2 k-e_{1}-e_{2}}^{j}=0
$$

and then we rearrange the prediction error into detail coefficients as follows:

$$
\begin{aligned}
& d_{k}^{1, j-1}:=\frac{1}{4}\left(e_{2 k-e_{1}-e_{2}}^{j}-e_{2 k-e_{2}}^{j}+e_{2 k-e_{1}}^{j}-e_{2 k}^{j}\right) \\
& d_{k}^{2, j-1}:=\frac{1}{4}\left(e_{2 k-e_{1}-e_{2}}^{j}+e_{2 k-e_{2}}^{j}-e_{2 k-e_{1}}^{j}-e_{2 k}^{j}\right) \\
& d_{k}^{2, j-1}:=\frac{1}{4}\left(e_{2 k-e_{1}-e_{2}}^{j}-e_{2 k-e_{2}}^{j}-e_{2 k-e_{1}}^{j}+e_{2 k}^{j}\right) .
\end{aligned}
$$

One can then easily check that $d^{1, j-1}, d^{2, j-1}$ and $d^{3, j-1}$ respectively correspond to detail coefficients in the horizontal, vertical and oblique directions, so that we have the same representation as in the orthogonal 
wavelet transform. This also implies that we should be able to use powerful encoders such as EZW [1] to encode the decomposition since these encoders are designed for such a quadtree structure.

\section{EXPERIMENTAL RESULTS}

In the previous section, we defined the prediction operator on O-cells using biquadratic polynomials based on a stencil either centered or shifted and on S-cells using piecewise polynomials. Our goal is here to investigate how different choices for prediction operator impact the NMR. To this end, we consider NMRs built either assuming the image is piecewise constant on each side of a straight line edge (the parameters of the edge being in that case computed using the ENO-EA strategy detailed in Section IV-A). The associated prediction will be denoted by $S R_{1}$ in the simulations, $S R$ standing for subcell resolution. We can also assume that the image is piecewise constant on each side of a curved-edge locally modeled by a second order polynomial (prediction denoted by $S R_{2}$ ) or finally by a biquadratic polynomial on each side of a curved-edge (prediction denoted by $S R_{3}$ ). The idea motivating the use of nonlinear prediction operators is to drastically reduce the number and the amplitude of detail coefficients generated by the presence of edges. We first illustrate this by studying the decay of detail coefficients on various synthetic images. Then, we investigate the NMR performance in terms of compression using an adapted version of EZW algorithm [1]. Finally, we show how our approach can be used for super resolution problems. Since most image processing algorithms are designed for $L^{2}$-normalized transform and since the studied NMR are $L^{1}$-normalized, we first define the renormalized representations as follows:

$$
\tilde{\mathcal{M}} v=\left(2^{J-1} v^{0}, 2^{J-1} d^{0}, \ldots, 2^{p-1} d^{J-p}, \ldots, d^{J-1}\right) .
$$

\section{A. Comparison of NMRs on Synthetic Images: Decay of Detail Coefficients}

In this section, we perform several numerical tests to illustrate the faster decay of the renormalized detail coefficients when one uses a nonlinear prediction rather than a linear one. Our goal is also to show how the edge model impacts the decay of the detail coefficients. For that purpose, we consider 3 different synthetic images (see Figure 6 first row), all of size $128 \times 128$, that is $J=2^{7}$. On the one hand, we consider linear predictions corresponding to the Haar basis (Haar prediction) and to the prediction

using the polynomial $p_{k}$ on each cell $C_{k}^{j-1}$ (Linear prediction) and on the other hand, the three different types of nonlinear predictions $S R_{1}, S R_{2}$ and $S R_{3}$ mentioned above. We depict on Figure 6 second row the decay of the amplitude of the renormalized detail coefficients (nonlinear decomposition is performed 


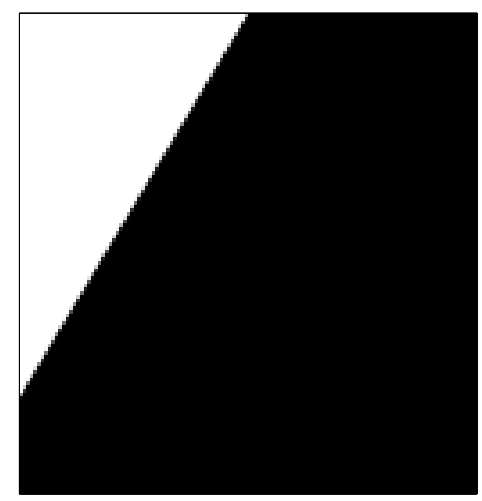

A

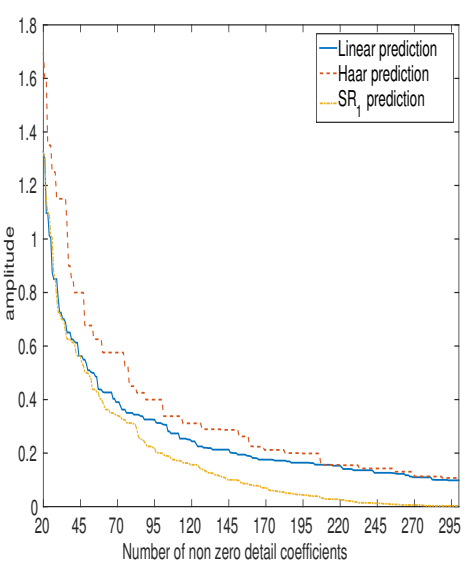

$\mathrm{D}$

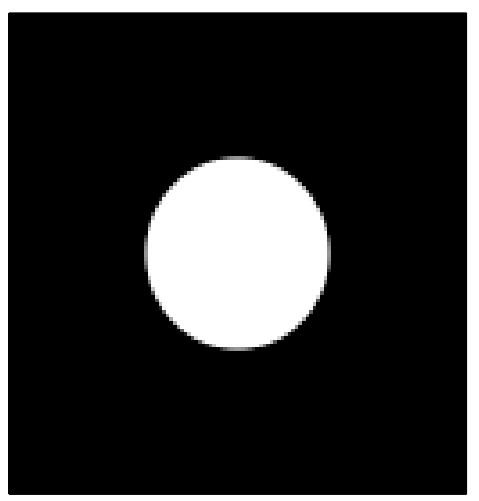

B

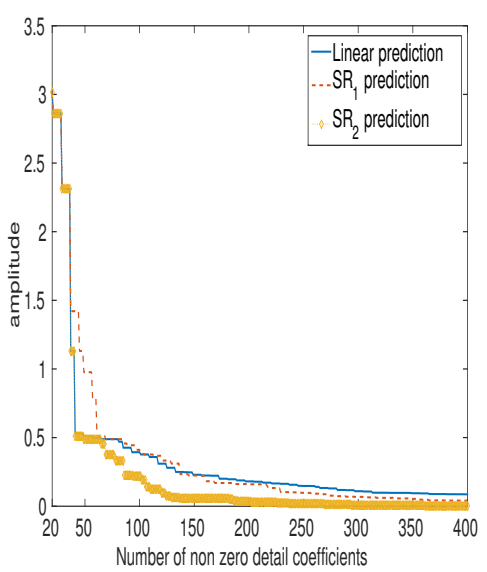

$\mathrm{E}$

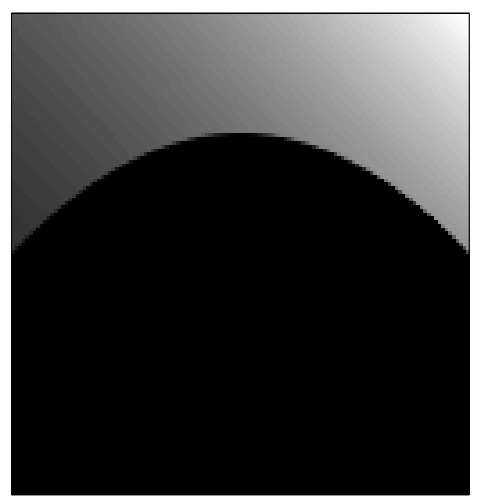

$\mathrm{C}$

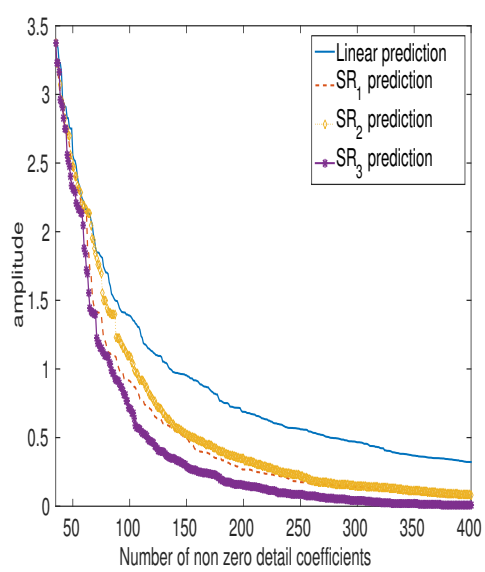

$\mathrm{F}$

Fig. 6. A: $128 \times 128$ image of a straight-line edge (i.e. averaged over cells of size 1/128 $\times 1 / 128$ ); B: $128 \times 128$ image of a circle (i.e. averaged over cells of size 1/128 $\times 1 / 128$ ); C: $128 \times 128$ image of a piecewise biquadratic model; D: decay of the renormalized detail coefficients using different linear and nonlinear methods on image A (depth of decomposition 3); E: idem as D but for image B; F: idem as D but for image C.

only at the first 3 levels of decomposition for $S R_{1}, S R_{2}$ and $S R_{3}$ techniques, linear decomposition being then performed at coarser scales). For the image of Figure $6 \mathrm{~A}, S R_{1}$ and $S R_{2}$ and $S R_{3}$ are the same, so we only compare linear predictions with $S R_{1}$ in that case. What is remarkable on Figure $6 \mathrm{D}$ is that linear and $S R_{1}$ prediction coincides for the first 20 or so coefficients: these coefficients are associated with levels smaller or equal to $J-4$. Then the decay of the detail coefficients for $S R_{1}$ is much faster and is controlled by the behavior of detail coefficients at levels larger or equal to $J-3$. It is important to notice here that nonlinear prediction does not create detail coefficients at level larger than $J-3$ having 


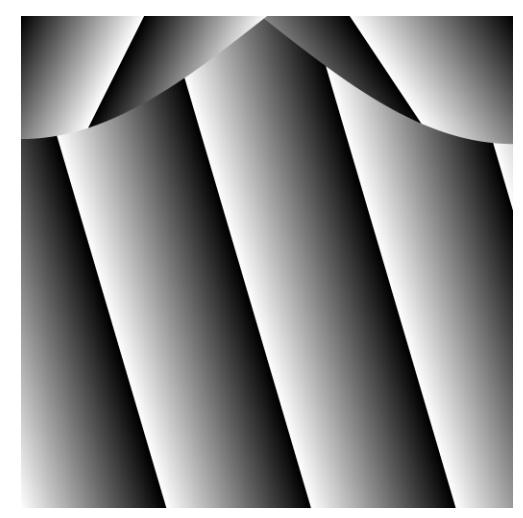

A

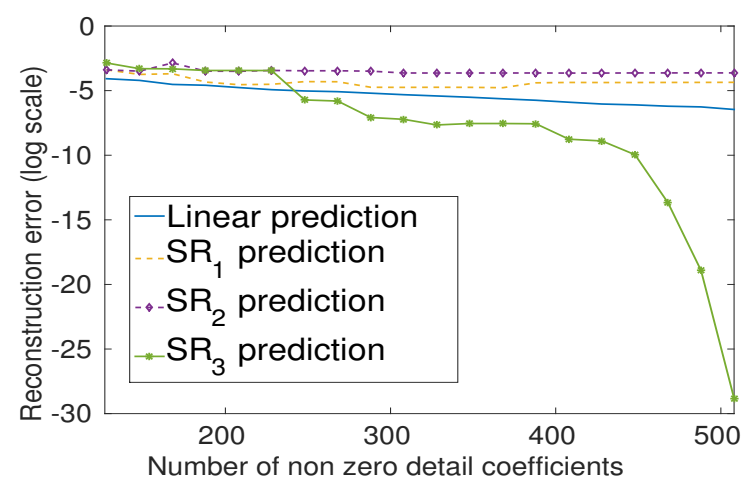

$\mathrm{C}$

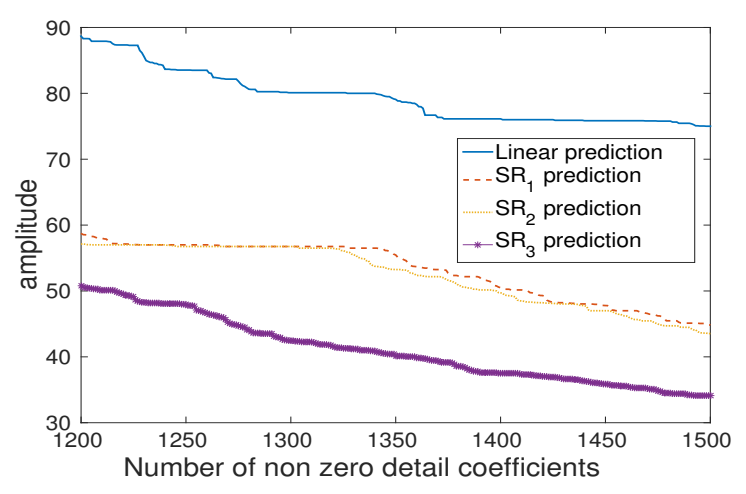

B

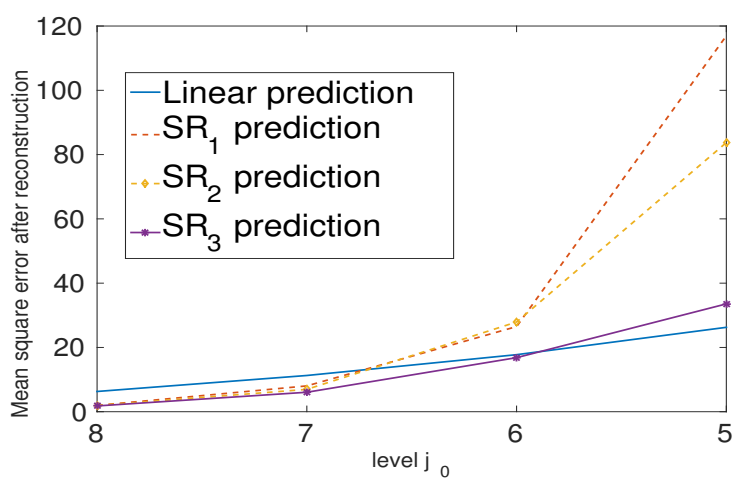

$\mathrm{D}$

Fig. 7. A: $512 \times 512$ geometric image containing linear, curvilinear edges and T-junction; B: decay of detail coefficients using linear and nonlinear methods computed on the finest three levels of decomposition for image A; C: Nonlinear reconstruction error corresponding to image of Figure for image A; D: mean square reconstruction error associated with super-resolution procedure using only the information available at level $j_{0}$ for image A.

an amplitude larger than that of the detail coefficients obtained at level smaller than $J-4$.

Then, for the image of Figure $6 \mathrm{~B}, S R_{2}$ and $S R_{3}$ are the same since $p_{a, k}$ and $p_{b, k}$ used by $S R_{3}$ reduce to constants in that case, so we only depict the results associated with $S R_{2}$. For such an image, the behavior of the different decompositions, depicted on Figure 6 E, are the same as previously: the first detail coefficients of the transforms are the same (corresponding to the level where the decomposition is linear) and then the decay is faster with $S R_{2}$ which is the only method to take into account the curvature of the edge. The bad behavior of $S R_{1}$ on this image indicates that it is worth taking into account the curvature of the edge when possible: the straight-line model on S-cells is a too crude approximation of the edge at the scales of study (some of which are thus larger than the critical scale $h_{c}$ mentioned in Section IV). 
Finally, for a piecewise biquadratic signal as the one of Figure $6 \mathrm{C}$, one notices again that the largest detail coefficients are the same for each type of decompositions. Then, as far as the remaining coefficients are concerned, to consider a biquadratic model on each side of the edge significantly improves the prediction as $S R_{3}$ behaves much better than other methods. Another illustration of the decay of the detail coefficients is given on Figure 7 B for the more sophisticated image of Figure 7 A, for which the advantage of using $S R_{3}$ to predict is again significant.

\section{B. Comparison of NMRs on Synthetic Images: Nonlinear Approximation}

Let us define $\tilde{v}^{N, J}$ the approximation of $v^{J}$ obtained by keeping the $N$ largest detail coefficients, and then $\varepsilon_{N}:=\left\|\tilde{v}^{N, J}-v^{J}\right\|_{2}$. The $L^{2}$ error obtained by keeping the $N$ largest detail coefficients of a $L^{2}$-normalized wavelet transform satisfies $\left\|\varepsilon_{N}\right\|_{2} \leq C N^{-s}$, and one obtains $s=1$ for bounded variation images [10]. Our goal is to illustrate here that a faster decay of the normalized detail coefficients entails a faster decay of the approximation error $\varepsilon_{N}$. As previously, for $S R_{1}, S R_{2}$ and $S R_{3}$ method we consider that the prediction is nonlinear at the finest three levels of decomposition and linear afterwards. We display $\varepsilon_{N}$ on Figure $7 \mathrm{C}$ for the image of Figure $6 \mathrm{C}$ corresponding to the different multiscale decompositions. We notice that the results are in accordance with the decay of the detail coefficients observed on Figure 6 $\mathrm{E}$ and the reason for such a behavior can be explained as follows. Despite S-cells are unknown and since the largest detail coefficients are at the coarsest scales, the algorithm first integrates detail coefficients belonging to these scales thus progressively reconstructs the set of S-cells while adding new detail coefficients. This remark is very important since it circumvents satisfactorily the problem of the absence of synchronization which can be stated as: there is no reason for S-cells to be the same at the encoding and decoding steps. Indeed, since the proposed NMRs do not create large coefficients at fine scale, S-cells are properly recovered leading to a faster decay of the reconstruction error. Furthermore, this study also shows that the edge parameters are accurately estimated even when all the detail coefficients are not available. Finally, we should mention that the reconstruction error also drastically decreases with all the other methods but for a much larger $N$.

\section{Application of NMRs to Super-Resolution Image Reconstruction}

Super-resolution is a class of techniques to enhance the resolution of an imaging system. In principle, the latter is limited by the diffraction limit which in short means that the image is lowpass filtered at the acquisition. This has the consequence that some high frequencies are not resolved. 
Transposed to our context, the diffraction limit means that the information on the image is not available at levels larger than some $j_{0}$. The challenge is thus to reconstruct an approximation of the original image $v^{J}$ as faithful as possible using only the coarse approximation $v^{j_{0}}, j_{0}<J$. The literature on superresolution techniques relative to image processing is huge and our goal is not to compare to existing techniques but to show the relevance of using nonlinear techniques in that context. For a review on super-resolution image reconstruction we refer the reader to [11] and the references therein.

The fact that nonlinear prediction operators lead to very few significant high frequency detail coefficients should naturally result in good behavior regarding super-resolution. Indeed, the knowledge of the high frequency detail coefficients should matter much less than when the linear prediction is used. We check this property by measuring the mean square error of the reconstruction process, i.e. $\frac{1}{2^{2 J}} \sum_{k}\left(\tilde{v}_{k}^{j_{0}, J}-v_{k}^{J}\right)^{2}$ where $\tilde{v}^{j_{0}, J}$ is the reconstructed image at level $J$ using only the information available at level $j_{0}$.

The reconstruction process is either linear or nonlinear and the results are depicted on Figure $7 \mathrm{D}$ for the image of Figure 7 A. To interpret the results, we note that since the studied image is $512 \times 512$, $J=9$ and we reconstruct the image using $v^{j_{0}}, j_{0}<J$. To use nonlinear prediction techniques appears to improve super-resolution performance for level $j_{0} \geq 7$. Furthermore, to consider a more accurate edge model, i.e. to use $S R_{3}$, leads to significantly lower reconstruction error compared with other studied nonlinear methods and also with linear method provided $j_{0} \geq 6$.

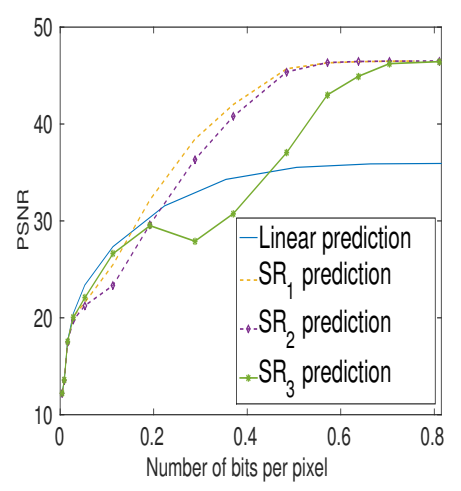

A

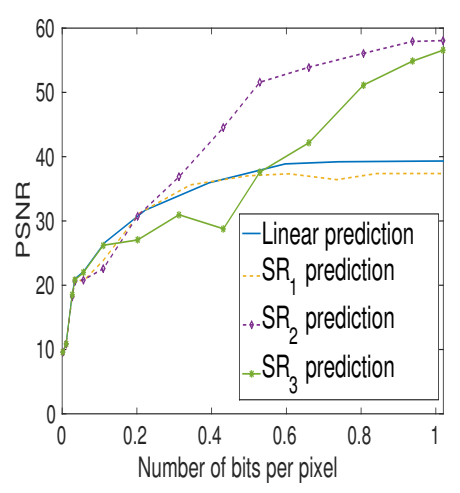

B

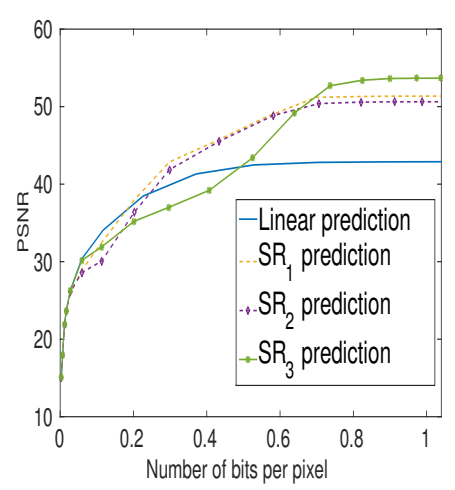

$\mathrm{C}$

Fig. 8. A: Compression results with EZW on image of Figure 6 A; B: idem for image of Figure 6 B; C: idem for image of Figure $6 \mathrm{C}$. 


\section{Image Compression}

We have just showed that the proposed NMRs have nice properties regarding nonlinear approximation and super-resolution performance. This could be explained by the fact that coarse levels of decomposition contain the information on the location of S-cells (i.e. see super-resolution performance) and, since nonlinear approximation integrates first coarse scales, S-cells are mostly recovered before the detail coefficients computed nonlinearly are added. Now, if one is interested in image compression, the problem is somehow different because not only partial information is available at the decoding step but also the latter is quantized. The questions are thus whether our algorithm can detect S-cells from a quantized transform and, if so, can the edge parameters be estimated accurately.

Since the proposed NMRs is associated with a quadtree structure it can be compressed using one of the most efficient compression algorithm, the so-called EZW (Embedded Zero-Tree) algorithm [1]. First, note that the renormalized NMR $\tilde{\mathcal{M}} v$ can be written under a matrix form following the same display of detail coefficients as the one used by orthogonal wavelet representation. We call this matrix $V^{J}$ in what follows. EZW algorithm, developed for wavelet transforms (supposed to be $L^{2}$-normalized therefore we use $\tilde{\mathcal{M}} v$ ), exploits the quadtree structure generated by the wavelet decomposition. The algorithm is based on progressive encoding: the data is compressed through multiple passes with increasing accuracy. EZW encoder builds zero-tree structures from the quadtree based on the observation that the wavelet coefficients decrease as $j$ increases.

In practice, we set the initial threshold for compression to $T_{0}=2^{\left\lfloor\log _{2}\left(\max \left|V^{J}\right|\right)\right\rfloor}$. The encoder then scans matrix $V^{J}$ using the Morton scan [12], compares each scanned coefficient with threshold $T_{0}$ and issues 'p', 'n', 'z' or 't' as outputs; if the magnitude of the scanned coefficient is larger than $T_{0}$, the output is 'p' if the coefficient is positive and 'n' otherwise, else the algorithm constructs a tree with the considered element as the root. If it is a zero-tree, i.e. the values at the nodes are all smaller or equal to the threshold, the output is 't', and 'z' (isolated zero) otherwise. EZW encoder assumes that there will be a very high probability that all the coefficients in a quadtree will be smaller than a certain threshold if the root is smaller than this threshold. One then encodes elements 'p' or 'n': one puts each of them in

a so-called 'subordinate list' associated with $\frac{3 T_{0}}{2}$ (resp. $-\frac{3 T_{0}}{2}$ ) when the associated coefficient is positive (resp. negative) with magnitude larger than $\frac{3 T_{0}}{2}$. After all the elements have been scanned, the threshold is set to $T_{0} / 2$ and the algorithm starts a new pass and finally stops after a number of passes corresponding to a predefined minimal value for the threshold. This procedure can be viewed as a bit-plane coding algorithm, the level of quantification depending on the number of passes. The just described encoding 
algorithm thus computes a sequence of quantized coefficients:

$$
\overline{\mathcal{M} v}=\left(2^{J-1} \bar{v}^{0}, 2^{J-1} \bar{d}^{0}, \ldots, 2^{p-1} \overline{d^{J-p}}, \ldots, \overline{d^{J-1}}\right) .
$$

To recover an approximation of the original image from the quantized decomposition, one writes the inverse operator, i.e. decoding step, as follows:

$$
\hat{v}^{J}=\tilde{\mathcal{M}}^{-1}\left(2^{J-1} \bar{v}^{0}, 2^{J-1} \bar{d}^{0}, \ldots, 2^{p-1} \overline{d^{J-p}}, \ldots, \overline{d^{J-1}}\right) .
$$

It is worth noting that due to the compression step, $\hat{v}^{j}$ is not equal to $v^{j}$ which implies that the prediction operator at the encoding step and at the decoding step will not be the same. This problem is known as the absence of synchronization between the encoder and the decoder. To avoid this problem, a possible choice would be to memorize the location of S-cells at each level during the encoding step and to reuse them in the decoding step [13]. However, this requires to allocate too many bits for that operation which deteriorates the compression results. On the contrary and similarly to what was shown previously in the application of NMRs to super-resolution and nonlinear approximation, we expect that the quantized decomposition will enable to recover automatically S-cells and estimate edge parameters provided the number of bits per pixel (bpp) is sufficiently high. In this regard, the compression results using EZW algorithm expressed in terms of the PNSR with respect to the bpp after compression is displayed on Figure 8. For the sake of consistency, we recall that the PSNR corresponds to the formula:

$$
P S N R=10 \log _{10}\left(\frac{Q^{2}}{\frac{1}{2^{2 J}} \sum_{k}\left(\hat{v}_{k}^{J}-v_{k}^{J}\right)^{2}}\right),
$$

where $Q$ is the maximum of the amplitude of the image coefficients. It is important to note here that for the different NMRs only the first level of decomposition is computed nonlinearly.

Looking at the results depicted on Figure 8, we see that when the image contains only a straight line edge $S R_{1}$ and $S R_{2}$ behaves similarly which means that $S R_{2}$ adapts to non curved edges (see Figure 8 A). Furthermore, when the edge is actually curved, better compression results are obtained by taking into account the curvature at the encoding step (compare $S R_{1}$ and $S R_{2}$ on Figure $8 \mathrm{~B}$ ). In these first two cases, we also notice that when one uses a more sophisticated edge model such as $S R_{3}$, the number of bpp to recover the edge parameters is more important than with $S R_{1}$ and $S R_{2}$. Finally, we notice on Figure $8 \mathrm{C}$ that $S R_{3}$ behaves better than $S R_{1}$ and $S R_{2}$ when the image is actually a piecewise biquadratic polynomial and provided enough bits are transmitted. We finally give an illustration of the compression performance of the proposed NMRs on the image of a convection flow (see Figure 9 A) for which we notice on Figure $9 \mathrm{~B}$ a better behavior of $S R_{3}$ compared with $S R_{1}$ and $S R_{2}$. 


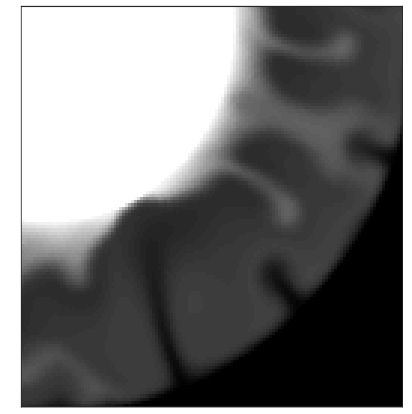

A

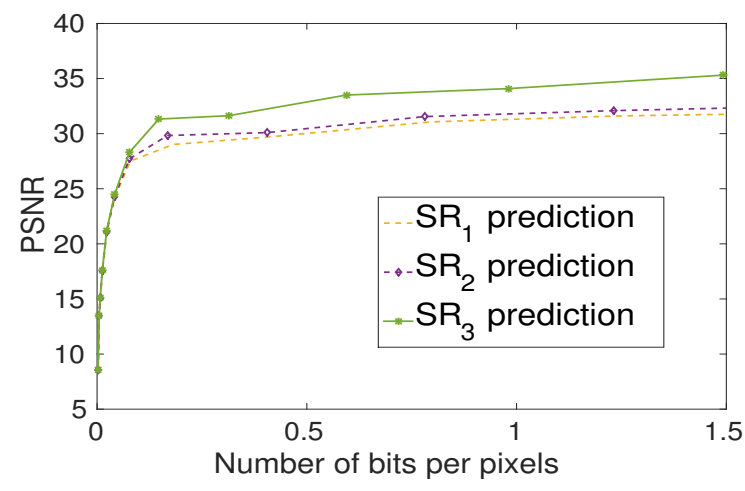

B

Fig. 9. A: Image of a convection flow, the heat source is at the top left; B: Compression results using the different NMRs

\section{CONCLUSION}

In this paper, we derived a new type of nonlinear multiscale representations based on nonlinear prediction operator in the cell-average framework. As the structure obeys the same quadtree structure as orthogonal wavelet transform compression algorithm such as EZW can be applied to the nonlinear multiscale representation. In this regard, we noticed significant improvement in terms of compression performance compared with the linear multiscale representations. Another application of the proposed nonlinear representations is on super-resolution for which we showed that accurate reconstruction of piecewise regular images could be achieved using an approximation of the image at a coarse resolution level.

\section{REFERENCES}

[1] Shapiro J., "Embedded image coding using zerotrees of wavelet coefficients," IEEE Transactions on Signal Processing, vol. 12, pp. 3445-3462, 1993.

[2] Taubman D., "High performance scalable image compression with ebcot," IEEE Transactions on Image Processing, vol. 9, pp. 1158-1170, 2000.

[3] E. Candes and D.L. Donoho, "New tight frames of curvelets and optimal representations of objects with piecewise $c^{2}$ singularities," Comm. on Pure and Appl. Mathematics, vol. 57, pp. 219-266, 2002.

[4] M.N. Do and M. Vetterli, “The contourlet transform: an efficient directional multiresolution image representation," IEEE Trans. Imag. Proc., vol. 14, pp. 2091-2016, 2005.

[5] E. Le Pennec and S. Mallat, "Sparse geometric image representations with bandelets," IEEE Transactions on Image Processing, vol. 14, pp. 423-438, 2005.

[6] R. Baraniuk, H. Choi, J. Romberg, and M. Wakin, "Wavelet-domain approximation and compression of piecewise smooth images," IEEE Trans. Im. Proc., vol. 15, pp. 1071-1087, 2006. 
[7] F. Arandiga, A. Cohen, R. Donat, N Dyn, and Mateï B., "Approximation of piecewise smooth images by edge-adapted techniques," Appl. Comput. Harmon. Anal., vol. 24, pp. 225-250, 2008.

[8] A. Harten, "Discrete multiresolution analysis and generalized wavelets," J. of App. Num. Math., vol. 12, pp. 153-193, 1993.

[9] A. Harten, B. Enquist, S. Osher, and S. Chakravarthy, "Uniformly high order accurate essentially non-oscillatory schemes iii,” J. Comp. Phys., vol. 71, pp. 231-303, 1987.

[10] A. Cohen, R. DeVore, P. Petrushev, and H. Xu, “Non-linear approximation and the space BV $\left(\mathbb{R}^{2}\right)$," American Journal of Mathematics, vol. 121, pp. 587-628, 1999.

[11] S. C. Park, M. K. Park, and G. K. Moon, "Super-resolution image reconstruction: A technical overview," IEEE Signal Processing Magazine, vol. 20, no. 3, pp. 21-36, 2003.

[12] V.R. Algazi and R.R.: Estes, "Analysis based coding of image transform and subband coefficients," in Proceedings of the SPIE, 1995, 2564, pp. 11-21.

[13] V. Chappelier and C. Guillemot, "Oriented wavelet transform for image compression and denoising," IEEE Trans. Image Process., vol. 15, pp. 2892-2903, 2006. 